\title{
La herencia pragmática de Gottlob Frege y Charles Sanders Peirce*
}

\author{
The pragmatic heritage of Gottlob Frege \\ and Charles Sanders Peirce
}

\section{A herança pragmática de Gottlob Frege e Charles Sanders Peirce}

Fecha de entrega: 5 de noviembre de 2014 Fecha de evaluación: 25 de noviembre de 2014 Fecha de aprobación: 15 de diciembre de 2014

César Augusto Vásquez García**

\section{Resumen}

El presente artículo muestra cómo los problemas de orden lógico estudiados por Frege y Peirce a finales del siglo XIX devienen en el desarrollo de la filosofía analítica y en la filosofía del lenguaje en el siglo $\mathrm{XX}$. En ese sentido, existe un hilo conductor que articula las propuestas reflexivas de los pragmatistas clásicos con los últimos desarrollos lingüísticos desde la semántica y la pragmalingüística en el marco de las consideraciones sobre los enfoques del lenguaje, entendido este

* El presente artículo hace parte de las reflexiones que el autor hace desde el curso de Semántica que imparte en la Facultad de Filosofía y Letras de la Universidad Santo Tomás, y que fueron socializadas en el simposio "De la analítica al (neo) pragmatismo", en el marco del V Congreso Colombiano de Filosofía realizado en la ciudad de Medellín en julio de 2014.

* Docente de la Facultad de Filosofía y Letras. Universidad Santo Tomás, Bogotá. E-mail: cesarvasquez@ usantotomas.edu.co. Coinvestigador del Grupo Fray Bartolomé de las Casas en la línea Historia de las Ideas en América Latina. 
como una red de significados construidos en contextos de enunciación específicos.

Palabras clave: lenguaje, pragmatismo, filosofía analítica, filosofía del lenguaje, Frege, Peirce.

\section{Abstract}

This article shows how the problems of logic order studied by Frege and Peirce in the late nineteenth century become in the development of analytical philosophy and the philosophy of language in the twentieth century. In this regard, there is a central strand which articulates the reflective proposal of the classical pragmatists with the latest linguistic developments from semantics and pragmalinguistics in the frame of the considerations about the approaches of language, understood this as a network of meanings built in specific formulation contexts.

Keywords: Language, pragmatism, analytical philosophy, philosophy of language, Frege, Peirce.

\section{Resumo}

O presente artigo pretende mostrar como os problemas de ordem lógica estudados por Frege e Peirce, no final do século XIX, proporcionaram o desenvolvimento da filosofia analítica e da filosofia da linguagem no século XX. Nesse sentido, existe um fio condutor que articula as propostas reflexivas dos pragmatistas clássicos com os últimos desenvolvimentos linguísticos desde a semântica e a pragmalinguística no marco das considerações sobre o enfoque da linguagem, entendido como uma rede de significados construídos em contextos de enunciação específicos.

Palavras-chave: Linguagem, pragmatismo, filosofia analítica, filosofia da linguagem, Frege, Peirce.

"El pragmatismo no resuelve ningún problema real, pero sí muestra que presuntos problemas no son problemas reales".

Charles Sanders Peirce 
"En esencia el pensamiento auténtico es herejía, trasgresión de las creencias, de los prejuicios y los hábitos que se han heredado por generaciones. (...) Así, en lugar de permitir la claridad sobre el mundo, hacen que éste se desdibuje dentro de un verbalismo, aparentemente mágico. Y el lenguaje, ese instrumento de la expresión y de la comunicación, es ahora un perverso juego que oculta la miseria en que se vive. El pensamiento pierde entonces su poder creador para convertirse en una sarta de sentencias que le ahorran al hombre la tarea de comprender...”.

Rubén Sierra Mejía. Lección de los doctorados Honoris Causa de la Universidad de Caldas

La idea básica que encierran los dos epígrafes anteriores remite a la consideración de pensar, primero, que existen pseudo problemas a lo largo y ancho del pensamiento filosófico envueltos en el ropaje de las palabras en una especie de verbalismo oscuro ${ }^{1}$ $\mathrm{y}$, segundo, que dichos pseudo problemas se pueden detectar y desdibujar a través de la conciencia de la heredad asumida desde el análisis, la reflexión y la autocriticidad ${ }^{2}$.

Todos somos conscientes de lo que hemos heredado de nuestros padres y abuelos y, aunque el hecho de que en ocasiones no nos sintamos complacidos con lo heredado, no impide reconocer que esta heredad hace parte de nuestra biografía. En este sentido, el título del presente texto asume el concepto de "herencia" desde la perspectiva del carácter autocrítico de la corriente de pensamiento pragmatista que deviene de las propuestas lógicas y epistemológicas de Gottlob Frege y Charles Sanders Peirce, sin que necesariamente se reconozca en ellos una filiación directa y explícita con

1 "El error de la filosofía, y el punto a partir del cual su imperativo de claridad habria empezado a degenerar en un prurito de oscuridad, consiste en haber pretendido apresar la realidad de las cosas directamente desde su limitada posición, haciendo ver que se trata de un punto de vista privilegiado, o incluso excluyente, para acceder a una verdad superior" (Faerna, 1996, p. 10).

2 "La primerisima lección que tenemos derecho a exigir que nos enseñe la lógica es la de cómo esclarecer nuestras ideas; y qué importante resulta, despreciada como es solo por mentes que tienen necesidad de ella. Saber lo que pensamos, ser maestros de nuestra propia significación, constituirá un fundamento sólido para un pensamiento grande e importante" (Peirce, 2012, p. 172). 
los desarrollos de la filosofía analítica ${ }^{3}$ en general, y de la filosofía del lenguaje en particular. De allí que nuestro interés recaiga en la percepción, tímida y cautelosa, de avisorar una arista, un intersticio en el devenir de la filosofía del lenguaje, esto es, el asunto de las relaciones de la lingüística como ciencia del lenguaje verbal y los desarrollos pragmatistas arropados bajo el ámbito de la filosofía analítica durante el siglo XX y parte del XXI a la luz de las ideas de quienes han sido asumidos por la tradición intelectual como los núcleos generativos de la reflexión contemporánea sobre las lógicas del lenguaje humano ${ }^{5}$.

Lo primero que debemos tener en cuenta es que Frege y Peirce eran contemporáneos (Peirce: 1839-1914; Freg: 1848-1925) y desarrollaron sus investigaciones en el campo de la lógica matemática, ciencia que en el siglo XIX tuvo enormes desarrollos conceptuales y metodológicos (baste con mencionar los trabajos de Cantor, Boole o Peano para entender el dinamismo de las teorías matemáticas en esta época). La filosofía del lenguaje o, si se prefiere, la semántica filosófica tal como lo sugiere Korta (2002, p. 346), nace de estos dos matemáticos involucrados en la fundamentación

3 "La denominación "filosofía analítica" a pesar de su extraordinaria difusión y de su indudable éxito, es un nombre vago e impreciso, pero conforma con claridad una tradición de investigación en filosofia, desarrollada predominantemente en inglés, que remonta sus orígenes inmediatos a los trabajos de Frege, Russell, Wittgenstein y el positivismo lógico del Círculo de Viena... Para la tradición analítica muchos problemas filosóficos vendrian a ser problemas básicamente lingüisticos, esto es, cuestiones que en muchos casos se disuelven, aclarando los enredos conceptuales en que nos han tenido nuestras palabras" (Nubiola, 2011, p. 112). "Aunque el término "análisis" se encuentre ya entre los griegos aplicado a la explicación de estructuras complejas mediante la identificación de sus elementos simples, la tesis fundamental del análisis filosófico de nuestro siglo tiene un sentido más preciso: el análisis es primordialmente un análisis lógico, esto es, consiste en el esclarecimiento de la forma lógica, de la estructura lógica subyacente al lenguaje" (Nubiola, 2011, p. 114).

4 "La filosofía del lenguaje resuelve problemas que, en todo caso, son de naturaleza lingüistica. Pero su importancia no reside solo en este simple hecho sino también en que ayuda a plantear con más precisión y nitidez los problemas típicos de las disciplinas filosóficas. Por ejemplo, la filosofía del lenguaje tiene su parte en el enfoque de uno de los problemas más acuciantes en la actualidad, el de la acción racional o de la racionalidad. La filosofía del lenguaje no podrá -ni deberá- decirnos a este respecto cuándo nuestras acciones son racionales o cuándo dejan de serlo, ni nos podrá enseñar a ser más racionales, pero en cambio si que podrá ayudar a dilucidar el concepto de racionalidad, pues es un concepto que utilizamos, a través de múltiples expresiones lingüisticas cotidianamente" (Acero, 2001, p. 16). "Esta teoria del significado tiene como representante más destacado a Searle (...) La estrategia central es considerar al lenguaje como un instrumento para hacer cosas y explicar lo que significan ciertas partes del lenguaje en función de su uso o finalidad" (Frápolli y Romero, citadas por Santamaria, 2014, p. 53).

5 "El modelo de Frege se refiere única y exclusivamente a signos lingüisticos, mientras que el de Peirce es un modelo de aplicación a cualquier cosa que funcione como signo, y en este contexto Peirce no está pensando exclusivamente en los signos lingüisticos" (Rivas, 2002, p. 60). 
lógica de la matemática, hecho al parecer curioso en el ámbito académico del siglo XIX, pues se daba por descontado que la matemática y la lógica eran disciplinas que no dialogaban con frecuencia entre sí. Por lo tanto, cuando Peirce y Frege asumieron su proyecto investigativo desde las fronteras de la lógica y la matemática, generaron cada uno por su cuenta la idea de buscar algo que se ha denominado "vocabulario mínimo" para proferir enunciados de carácter lógico que permitieran claridad y certeza a la hora de dar cuenta de las abstracciones matemáticas, tal como lo expresara posteriormente Bertrand Russell:

Pero lo que había sido demostrado era algo independiente en absoluto de la metafísica; algo que queda mejor establecido en términos de "vocabularios mínimos". Entiendo por "vocabulario mínimo" aquel que en ninguna palabra puede ser definida en términos de otras. Todas las definiciones son teóricamente superfluas y, por consiguiente, cualquier ciencia puede expresarse en su totalidad mediante un vocabulario mínimo de tal ciencia. Peano redujo el vocabulario especial de aritmética a tres términos, Frege y los Principia Mathematica sostuvieron que incluso estos eran innecesarios, y que el vocabulario mínimo de matemáticas es el mismo de la lógica (Russell, 1994, vol. I, p. 113).

Ya es claro para nosotros, entonces, que las ciencias en general, y la matemática en particular, se proponen (se escriben y se leen) a través de gramáticas específicas que asumen reglas determinadas para construir su aparato conceptual, lo que deviene para finales del siglo XIX y principios del siglo XX en el reto mayúsculo de diseñar un vocabulario mínimo, una enciclopedia básica y fundamental para hacer con palabras lo que se considera como estatuto epistemológico de las disciplinas. Es en este sentido en que se habla de un "giro lingüístico" de la filosofía

6 En la siguiente cita de Rorty, retomada por Jaime Nubiola, se comprende mucho mejor el sentido de lo que significa esta expresión: "El giro lingüistico de la filosofia se caracteriza tanto por la concentración de la atención en el lenguaje como por la tendencia a abordar los problemas filosóficos a partir de la forma en que aparecen en el lenguaje... El origen de este giro se sitúa comúnmente en los trabajos del matemático alemán Gottlob Frege (1848-1925), quien aspiraba a encontrar un lenguaje conceptual que expresará perfectamente la estructura de los razonamientos de la matemática. Frege estaba convencido de que hay un paralelismo entre pensamiento y lenguaje, de que el lenguaje es, por así decir, la expresión sensible del pensamiento. A Frege le interesaba el pensamiento, no tanto el lenguaje en sí mismo; se preocupó del lenguaje en la medida en que afecta a la expresión del pensamiento para eliminar todos aquellos elementos del lenguaje que resultaban irrelevantes o incluso eran engañosos para la expresión ingenua del pensamiento. Su estrategia para analizar el pensamiento 
Ahora bien, aclaremos como lo plantea Moya -quien fuera presidente de la Sociedad Española de Filosofía Analítica- que el interés de Frege por la filosofía del lenguaje es sobre todo semántico a partir del acento en las categorías de significado, denotación y verdad:

Pues la distinción entre las condiciones de verdad y valor de verdad es una aportación esencial de Frege a la teoría del significado. (...) La oración, y no la palabra aislada es para Frege una unidad mínima del significado. Solo la oración tiene condiciones de verdad, expresa un pensamiento por cuya verdad o falsedad podemos preguntar inteligiblemente. Una palabra solo tiene significado en el contexto de una oración. Este es el llamado "Principio del Contexto" (2011, p. 6).

De hecho, Dummett lo reitera así:

According to the conception of language as a vehicle of thought, this explanation must embody an account of what it is to have the concepts expressible in the language; and Frege, who originated this new approach, gave the outlines of an explanation of this kind. (...) I am not here concerned with the particular features of Frege's theory, but only with the general line of approach to the philosophy of language of which it was the earliest example. Frege's theory was the first instance of a conception that continues to dominate the philosophy of languaje, that of a theory of meaning for a specific language. (...) The conception of mastery of a language as consisting in the implicit knowledge of a theory of meaning is just as much in accordance with our original notion that what makes the utterances of a speaker to be expressions of thought is a piece of internal equipment that he has, namely his general understanding of the language, as was the conception of language as a code (Dummett, 1996, pp. 99-101).

En ese mismo sentido, Russell y el Wittgenstein del Tractatus asumieron con Frege (se suele situar a "Sobre sentido y referencia" de Frege -1892- como la obra pionera en la formulación de esta problemática) la tarea de develar la lógica de los enunciados 
formalizados en oraciones ${ }^{7}$, estableciendo las relaciones entre sentido y referencia ${ }^{8}$ que posteriormente serán objeto de análisis por partes de Wittgenstein, Strawson, Kripke, Domellan, Putnam, Davidson y Quine, entre los más destacados filósofos del lenguaje. Es por ello que Dummett considera a Frege como el fundador de esta corriente filosófica:

En la medida en que hizo de la filosofía del lenguaje la disciplina filosófica fundamental, desplazando la teoría del conocimiento del lugar de privilegio que había ocupado en la modernidad a partir de Descartes. La primacía de la filosofía del lenguaje sería, pues, para Dummett la característica distintiva de la filosofía analítica. (Moya, 2011, p. 3).

Sin embargo, Méndez en el artículo "Pragmática sin pragmatismo" plantea, a diferencia de lo que afirma Richard Rorty, que no fueron Frege y Wittgenstein los que inicialmente dieron el giro lingüístico de la filosofía al señalar como prioridad las posibles relaciones del lenguaje con los objetos del mundo, antes que pensar en el fenómeno de la representación mental del mundo a través del lenguaje. En ese sentido "fue Peirce el que ya antes había reformulado la problemática kantiana en los términos del pragmatismo" (Méndez, 2009, p. 182), hecho ampliamente conocido por la historia del pensamiento norteamericano a finales del siglo XIX:

According to William James, Peirce introduced pragmatism into philosophy in an 1878 article "How to Make Our Ideas Clear". James wrote this in "What

7 "Frege partía de la concepción de que el lenguaje ordinario no ofrecia garantía alguna de seguridad: las ambigüedades y vaguedades de que estaba aquejado enmascaraban lo que él denominaba begriffliche inhalt (contenido conceptual) de las oraciones, aquello que servia de soporte a las inferencias" (Valdés, 1998, p. 14).

8 "En el empleo ordinario del lenguaje para formular enunciados, hacer preguntas, etc., se ha de asumir que todo signo completo y distinto posee a la vez sentido y referencia. La referencia es un objeto de algún género, pero no necesariamente un objeto físico perceptible; pues Frege se opuso siempre tenazmente a la tesis de que únicamente existe lo que puede ser percibido, incluyendo expresamente entre sus objetos cosas tales como números, lugares, instantes, o periodos de tiempo. El sentido, por su parte, es un aspecto bajo el que cabria considerar a nuestro objeto. No se trata en modo alguno de una idea, si por tal entendemos una imagen $u$ otro acaecimiento psíquico privativo de un sujeto" (Kneale, 1972, p. 458). "Para toda expresión, o para la mayoría de ellas, vale decir que hay una relación entre aquella y algún objeto $u$ objetos correspondientes -la relación a la que Frege llama Bedeutung- y otra entre la expresión y su Sinn. Para traducir estos términos alemanes se han hecho diversas propuestas: para Bedeutung, referencia (que es la que nosotros adoptamos), denotación o nominatum; para Sinn, sentido, o, simplemente, significado (que es la que elegimos por las razones que podrán verse)" (Christensen, 1968, p. 148). 
Pragmatism Means", Lecture Two of his 1908 book, Pragmatism. According to James, the idea lay dormant for twenty years until he, James, brought it forward in an address at the University of California in 1898. This section will first look at the key ideas in Peirce's "How to Make our Ideas Clear", next sketch the sources of the idea in Peirce's early background, and then consider Peirce's 1903 interpretation of Pragmatism, including his differences with James (Mullin, 2007, p. 121).

Pero miremos qué es el pragmatismo peirceano ${ }^{9}$ partiendo del hecho de que ni siquiera su colega y amigo James pudo comprender a cabalidad su propuesta epistemológica, tal como lo señala Aliseda:

El pragmatismo es un método filosófico de reflexión que tiene como propósito aclarar las ideas y es guiado en todo momento por los fines de las ideas que analiza, ya sean estos puramente práctico o del pensamiento. Se concibe como un método en lógica más que un principio metafísico (2003, p. 262).

Así las cosas, el pragmatismo se define como un método de reflexión lógico para la filosofía ${ }^{10}$. Lo paradójico es que el Peirce matemático (recordemos que heredó más que la pasión, la disciplina del pensamiento matemático del profesor de Harvard Benjamin Peirce, lo que nos hace acordar que a veces la heredad no siempre nos hace felices) supedita la lógica a la matemática, tal como lo manifiesta en el siguiente texto:

No me parece que la matemática dependa en algún modo de la lógica. La matemática razona, desde luego. Pero cuando el matemático vacila o yerra en su razonamiento la lógica no puede acudir en su ayuda. Aún más fácilmente cometería el matemático errores análogos y otros más en la lógica. Por el

9 "The essential characteristic of pragmatism abides in the conviction that our actions follow from our beliefs. The only propositions that we really believe are those that we are willing to act on. This philosophy produced as its main outcome, a worldview that is cientific without being materialistic and reductionist, and which gives a proper place to the communal and spiritual aspects of human existence" (Mullin, 2007, p. 12).

10 "Al enunciar Peirce la máxima pragmática como un "evangelio lógico" -sus colegas de entonces y sus criticos de hoy en día- no entendieron quizá que la noción de lógica en Peirce es algo mucho más amplio de lo que incluso concebimos en la actualidad. Para él, todo pensamiento se realiza en signos y a su vez todo razonamiento consiste en una inferencia lógica que tiene como finalidad la adquisición de creencias que conlleva a la creación de nuevos hábitos, ya sean estos epistémicos o de acción" (Aliseda, 2003, p. 270). 
contrario, lo que sí creo es que la lógica no puede conseguir la solución de sus problemas sin un amplio uso de la matemática. En realidad, toda la lógica formal es simplemente matemática aplicada a la lógica (Peirce, 1994, vol. 5, p. 161).

Peirce afirma que el problema del pragmatismo no es más que el problema de la lógica de la abducción ${ }^{11}$, lógica que rompe con los modos tradicionales de hacer ciencia, pues atribuye al pensamiento abductivo un valor hasta ahora insospechado que desbalancea los métodos inductivo y deductivo a la hora de conocer un objeto de estudio en cualquiera de los campos disciplinares conocidos hasta el momento. En efecto, desde esta lógica abductiva en Peirce "están presentes muchas de las características distintivas de la filosofía analítica tales como su énfasis en el análisis lógico y en el respeto por la coherencia...” (Méndez, 2009, p. 186); idea que queda condensada en la conocida "máxima pragmática peirceana": "It appears, then, that the rule for attaining the third grade of clearness of apprehension is as follows: Consider what effects, that might conceivably have practical bearings, we conceive the object of our conception to have. Then, our conception of these effects is the whole of our conception of the object"12.

La máxima pragmática de Peirce en principio no es más que una regla metodológica para el análisis del significado en la medida en que intenta aclarar "el contenido real de los conceptos empíricos mediante su conexión con la acción posible" (Faerna, 1996, p. 11). Con esto se propone al pragmatismo como un método filosófico más que como una mera filosofía de la vida, diferenciándolo así del pragmatismo de James: "Se verá (del enunciado original) que el pragmatismo no es una concepción del mundo (Weltanschauung) sino un método de reflexión que tiene como propósito reproducir ideas claras (CP. 5.13)" (Aliseda, 2003, p. 268).

En su estudio titulado "Scholarship on the relations between Ludwig Wittgenstein and Charles S. Peirce", el experto español en la obra peirceana Jaime Nubiola argumenta la existencia de un hilo argumentativo entre la concepción filosófica de Peirce

11 "... La abducción parece ser tanto un acto de intuición como uno de inferencia", como lo ha propuesto Anderson 1986), quien sugiere un doble aspecto en la abducción, el intuitivo y el racional" (Aliseda, 2003, p. 264).

12 "Considera cuáles son esos efectos que pudieran concebirse que tienen un alcance práctico que percibes que el objeto de tu concepción tiene. Entonces, tu concepción de esos efectos es la totalidad de la concepción del objeto" (Aliseda, 2003, p. 267). 
y el Wittgenstein de las Investigaciones filosóficas. Así, "resulta plausible afirmar que la vena pragmática del segundo Wittgenstein es de naturaleza peirceana desde el momento en que Wittgenstein leyó a James y fue ampliamente influenciado por Ramsey, quienes a su vez tuvieron contactos muy directos con Peirce" (Nubiola, citado por Méndez, 2009, p. 193).

Lo que aquí nos importa es insistir en que existen unos vasos comunicantes entre la primera filosofía del lenguaje en Frege y en Peirce, ya sea desde el horizonte lógico o desde el horizonte pragmático, y la filosofía analítica propiamente dicha en Russell y Wittgenstein, cuyo centro de interés es el lugar que el lenguaje ocupa con respecto al resto de dominios en filosofía y de la ciencias. Sin embargo, es evidentemente que, aunque la filosofía analítica ha prestado siempre una atención privilegiada al lenguaje, no se limita al análisis lingüístico, aunque "la filosofía analítica reconoce, sin duda alguna, un lugar especial a la filosofía del lenguaje" (Korta, 2002, p. 342).

Es claro que un supuesto común a los representantes del análisis clásico es un atomismo semántico moderado o enfoque sintacentrista, entendido como la tesis según la cual la unidad mínima del sentido es la oración (Moya, 2011, p. 11). No obstante, los problemas fregeanos clásicos que se revisan desde la segunda mitad del siglo XX asumen perspectivas pragmáticas que desbordan los análisis sobre el significado a finales del siglo XIX. Un ejemplo de ello es que para la época de Frege y de Peirce el tipo de lingüística que se realizaba no despertaba el interés de lógicos y filósofos del lenguaje y "no porque los lingüistas no estuvieran en esa misma época trabajando cuestiones semánticas, sino por el carácter casi exclusivamente histórico de sus investigaciones" (Nubiola, 1997, p. 5).

Esta concepción sintacentrista se ve reforzada entonces por los estudios estructuralistas del lenguaje en sus versiones chomskianas que consideraban todavía que el centro de las investigaciones lingüísticas estaban ubicadas práctica y exclusivamente en el horizonte del análisis oracional, en donde el componente semántico es subsidiario y complementario del sintáctico. En ese sentido, "el énfasis de Chomsky en la pureza de la sintaxis cuadraba poco con la preocupación de los filósofos por los aspectos del lenguaje más semánticos y pragmáticos" (Nubiola, 1997, p. 5). Sin embargo, solamente en la medida en que la semántica y la pragmalingüística fueron concibiéndose como ciencias autónomas de la lingüística general que asumen los enunciados lingüísticos en contextos específicos de enunciación, se empieza a reconocer "que fueron Frege y Russsell, por un lado, y Austen y Grice, por otro, los que, sin llegar a construir 
ciertamente teorías sistemáticas y empíricas de las lenguas naturales, cimentaron los fundamentos de las teorías semánticas y pragmáticas actuales" (Korta, 2002, p. 353), idea que es corroborada por Santamaría cuando dice:

La filosofía analítica, iniciada en Frege, Moore, Russell y el mismo Wittgenstein, parte del deseo logicista de hacer de la filosofía un saber cercano a la ciencia, desde la búsqueda de un lenguaje lógicamente perfecto. Este primer florecimiento no se quedó allí. Todo lo contrario: estos primeros desarrollos permitieron el surgimiento de la filosofía del "lenguaje ordinario", del uso y las relaciones, no de los signos entre sí, o de los signos con sus significados, sino de los signos con sus usuarios, y los usuarios con su contexto, es decir, el lenguaje tendrá un carácter constitutivamente social, donde las creencias son productos colectivos y se resuelven en el contexto social... (2014, p. 47).

Así, la semántica y la pragmalingüística consiguen en los últimos treinta años "emanciparse" de la filosofía del lenguaje, y empiezan a desarrollar sus teorías de manera autónoma gracias a los aportes de Austen, Searle y Grice: la teoría de los actos de habla, tras ser re-elaborada por Searle (1969), y la pragmática griceana, con importantes desarrollos como los de Bach y Harnish (1979), junto con nuevas teorías post-griceanas como la teoría de la pertinencia (Sperber y Wilson, 1986), han hecho también que la pragmática haya dejado de ser terreno exclusivo de filósofos y pertenezca a los programas de investigación de los lingüistas (Korta, 2002, p. 349).

Finalmente, retomemos las palabras provocadoras de Korta para cerrar provisionalmente esta re-visión problemática de la relación entre filosofía del lenguaje, filosofía analítica y pragmatismo.

Las resistencias a entender la filosofía del lenguaje como filosofía de la Lingüística provienen, creo yo, de dos tipos de preocupaciones. La primera, que esta concepción niega a la filosofía del lenguaje la posibilidad de generar verdades substantivas acerca del lenguaje. La segunda, que obliga al filósofo a formarse en una gran variedad de disciplinas, la mayoría de las cuales han alcanzado un grado de sofisticación conceptual y notacional impenetrable (Ibíd., p. 354).

Es claro, entonces, que desde la mitad del siglo XX los filósofos del lenguaje y los lingüistas han abordado el problema del lenguaje desde perspectivas autónomas pero a la vez complementarias; en esa medida es pertinente aseverar que "la filosofía del 
lenguaje no ha sido filosofía de la lingüística", aunque las propuestas teóricas desde Frege y Peirce han estado cimentadas en las lógicas proposicionales que subyacen a los enunciados lingüísticos. Por ello:

Es razonable concluir que la filosofía del lenguaje no ha sido filosofía de la Lingüística; sin embargo, ha sido precisamente la filosofía del lenguaje la que ha establecido los fundamentos de dos de las ramas principales de la Lingüística: la Semántica y la Pragmática (Ibíd., p. 347).

Lo cierto es que hoy en día "varios de los filósofos que se consideran como analíticos serían muy renuentes a aceptar que las cuestiones que les preocupan puedan resolverse mediante el análisis lógico-lingüístico"; Moya señala:

La filosofía analítica ha sido y es en la actualidad un movimiento de gran vitalidad, en cuyo seno han sido y siguen siendo cultivadas todas las disciplinas filosóficas clásicas, además de la filosofía del lenguaje, y que ha generado, además, nuevos campos de investigación (2011, pp. 4-5).

Así, la filosofía analítica actual a través de su rigurosidad y claridad metodológica asume la responsabilidad de decantar el pensamiento vehiculado por las palabras en su empeño por asumir la heredad proveniente de las investigaciones lógicas de Frege y Peirce, para a su vez exigir que las construcciones de los discursos que dicen sobre las cosas del mundo estén en dirección de continuar la tradición crítica de la filosofía occidental, desarrollándola en nuevas direcciones, reservando el compromiso de dicha tradición con la razón y la búsqueda racional de los argumentos surgidos del esfuerzo de la persistencia (peirceistencia) y la perseverancia (peirceverancia) por encontrar el valor de la coherencia lógica más que el valor de la verdad ${ }^{13}$, tal como lo expresara el mismo Peirce:

Un hombre debería amar y reverenciar al genio de su método lógico como a su novia, a la que ha escogido de entre todo el mundo. No es necesario que

13 "En primer lugar, no desearias estudiar lógica a menos que pretendieses razonar; $y$, sin duda, sostienes que el propósito del razonar es el de verificar la verdad. Parece asi que perteneces a la secta que mantiene que hay algo así como la verdad. ¿No deberias, entonces, ponerte de acuerdo precisamente sobre el alcance de esta tu opinión, y, también, sobre qué razón hay para mantenerla?" (Peirce, 1988, p. 335). 
desprecie a las otras; al contrario, puede honrarlas profundamente, y al hacerlo solo honra aún más a la suya propia. Pero ella es la que ha escogido, y sabe que tenía razón al elegirla. Y habiéndola elegido, trabajará y luchará por ella, y no se quejará de que haya golpes que encajar, sino que esperará que haya otros tantos y tan duros por dar, y se esforzará por ser el digno caballero y campeón de ella, de la llama de cuyos esplendores saca su inspiración y su valentía (2012, p. 171).

\section{Referencias}

Acero, J., Bustos, E., y Quesada, D. (2001). Introducción a la filosofía del lenguaje. Madrid: Cátedra.

Aliseda, A. (2003). Abducción y pragamati(ci)smo en Charles S. Peirce. En Cabanchik, Samuel y otros. El giro pragmático en la filosofía, 261-272. Barcelona: Gedisa.

Christensen, E. (1968). Sobre la naturaleza del significado. Barcelona: Labor.

Dummettt, M. (1996). What do I know when I know a langage? En The seas of langage, 94-105. Oxford: Clarendon Press.

Faerna, A. (1996). Introducción a la teoría pragmatista del conocimiento. Madrid: Siglo XXI.

Frege, G. (1996). Escritos filosóficos. Barcelona: Crítica.

Haack, S. (2001). Viejo y nuevo pragmatismo. En Diánoia, 46(47), 21-59. México, D.F.: UNAM-FCE.

Kenny, A. (1997). Introducción a Frege. Madrid: Cátedra.

Kneale, W. (1972). El desarrollo de la lógica. Madrid: Tecnos.

Korta, K. (2002). Hacer filosofía del lenguaje. En Revista de filosofía, 27(2), 337-359. Madrid: Universidad Complutense de Madrid.

Méndez, G. (2010). Pragmática sin pragmatismo. En Discusiones Filosóficas, 11(17), 181-203. Manizales: Universidad de Caldas. 
Moya, C. (2011). La evolución de la filosofía analítica. Sapientia, 67, 229-230. Disponible en http://bibliotecadigital.uca.edu.ar/repositorio/revistas/raicespragmatica-filosofia-analitica-nubiola.pdf

Mullin, R. (2007). Peirce and the Origin of Pragmatism. En The soul of classical American philosophy. The ethical and spiritual innsights of William Jemes, Josiah Royce, and Charles Sanders Peirce, 119-126. New York: State University of New York

Nubiola, J. (1997). Renovación en la filosofía del lenguaje: hacia una mejor comprensión de nuestras prácticas comunitarias. Rev. Logop., Fon., Audiol., 17(1), 3-10. Disponible en http://bibliotecadigital.uca.edu.ar/repositorio/revistas/ raicespragmatica-filosofia-analitica-nubiola.pdf

. (2011). Raíces pragmáticas de la filosofía analítica. Sapientia, 67, 229-230. Disponible en http://bibliotecadigital.uca.edu.ar/repositorio/revistas/raicespragmatica-filosofia-analitica-nubiola.pdf

Nubiola, J., y Zalamea, A. (2006). Charles Sanders Peirce. Razón e invención del pensamiento pragmatista. Barcelona: Anthropos.

Peirce, Ch. (1994). La esencia de la matemática. En Newman, James. SIGMA. El mundo de las matemáticas, (5). Barcelona: Grijalbo.

. (1988). ¿Por qué estudiar lógica? En El hombre, un signo, 332-391. Barcelona: Crítica.

. (2012). Cómo esclarecer nuestras ideas. En Obra filosófica reunida, tomo I (1867-1893). México, D.F.: Fondo de Cultura Económica.

(2012). La fijación de la creencia. En Obra filosófica reunida, tomo I (18671893). México, D.F.: Fondo de Cultura Económica.

Rivas, M. (2002). Signo, mediación y realidad. Una lectura de G. Frege y C.S. Peirce. En Agora -Papeles de Filosofía, 21(2), 49-72. Santiago de Compostela: Universidad Santiago de Compostela.

Russell, B. (1994). Mi desarrollo intelectual. En Newman, James. SIGMA. El mundo de las matemáticas, (1). Barcelona: Grijalbo. 
Santamaría, F. (2014). Esplendor, miseria y resplandor de la filosofía analítica. De la analítica al neopragmatismo. En Cardona, Porfirio y Santamaría, Freddy (directores). Estética analítica: entre el pragmatismo y el neopragmatismo, 19-63. Medellín: Universidad Pontificia Bolivariana.

Valdés, L. (1998). Introducción. En Frege, Gottlob. Ensayos de semántica y filosofía de la lógica, 11-45. Madrid: Tecnos. 